\title{
تحليل التقابل بين اللغة الفصحى واللغة العامية المصرية من الناحية الصوتية في غناء عمرو دياب
}

\author{
Mamnunah \\ (IAIN Madura/mamnunah04@gmail.com)
}

\begin{abstract}
Egyptian Arabic Âmiyah and Arabic Fushâ have similarities and differences in terms of consonant and vowel sound, the case of similarities and differences of those phonological aspects were found in Amr Diab's song. This research is intended to know the similarities and the differences of consonant and vowel sounds between Egyptian Arabic Âmiyah and Fushâ in Amr Diab's songs. The contrastive analysis was utilized to identify the differences and the similarities of both languages in the subject. The results revealed the similarities of consonant sounds between Egyptian Arabic Âmiyah and Arabic Fushâ found in the

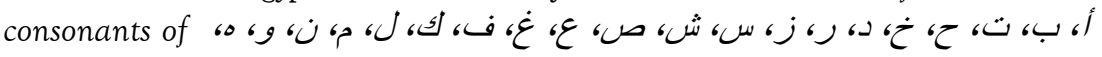
s, while the differences are in the consonant sounds of $\dot{j}$ which is pronounced as sound the same as!. Then, the similarities in vowel sounds between Arabic Fushâ and Egyptians Arabic Âmiyah are sounds of fathah, dhommah, kasrah thowilah \{long\} and fathah, dhommah, kasrah qashirah \{short\}, and the differences of vowel sounds are between the sounds of fathah and kasrah $\{\hat{e}\}$ also vowel of fathah and dhommah $\{\hat{o}\}$.
\end{abstract}

Keywords:

Contrastive Analysis, Arabic Fushâ, Egyptian Arabic Âmiyah, Phonology

DOI: $10.19105 /$ ojbs.v12i2.2014

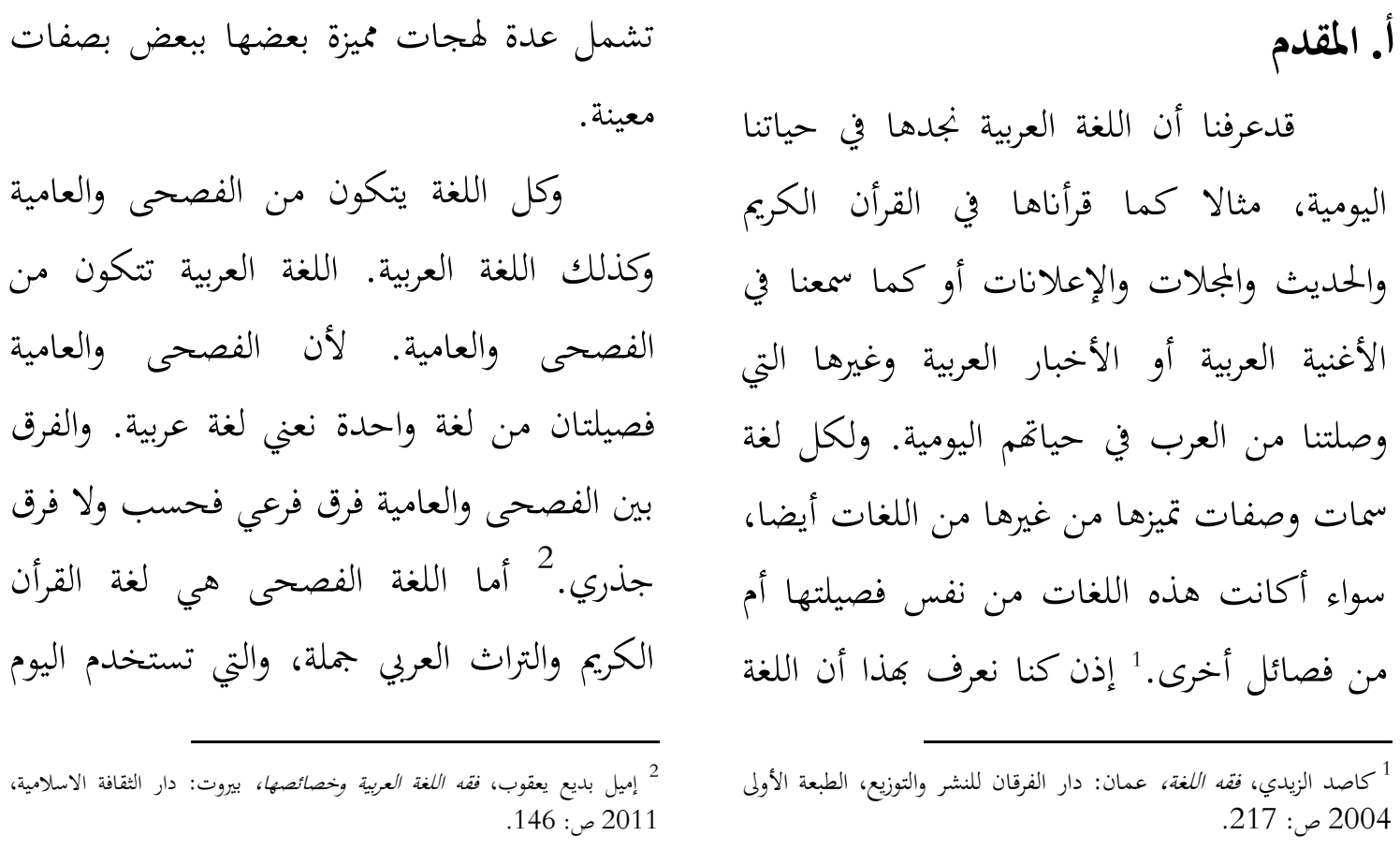


خخصوصة، بل هي مزيج من لهجات نجد وما

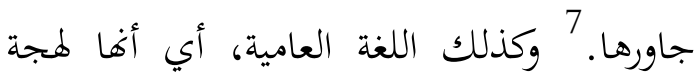
أيضا، المثال اللهجة لليمن، للحجاز، للسعودي وما إلى ذلك، ولكن تستخدم في حالة غير رسمية. وفي الواقعية نجد ما في بلادنا إندونيسيا من التعلم والتعليم والعلوم الذين بها نفهم معاني

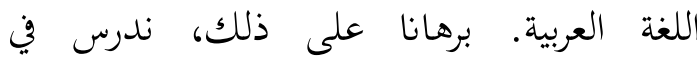
إندونيسيا من علم الصرف والنحو والبلاغة والمنطق وغيرها حتى نفهم معاني اللغة العربية عميقا من خلال تلك العلوم كلمة فكلمة وجملة

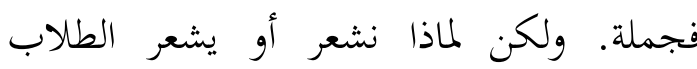

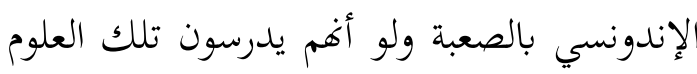
حينما يسمعون الأفلام والأخبار والإعلانات والأغاني العربية وغير ذلك في التلفاز أو الإذاعات أوغيرهما التي يستخدمون اللغة العامية، لاسيما

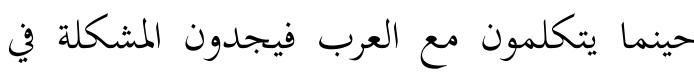

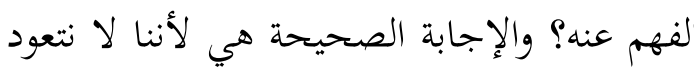

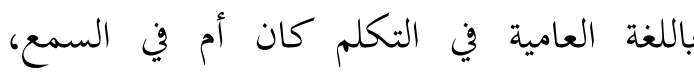
وبجانب ذلك، نستخدم في إجراء التعليم اللغة

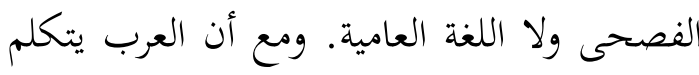

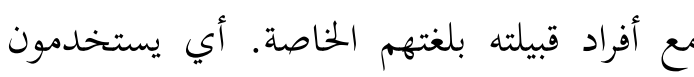
العرب اللغة العامية الخاصة لألة الاتصال بينهم.

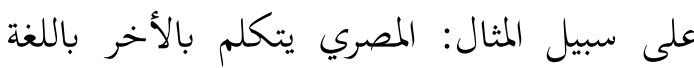
العامية المصرية، والسعودي يتكلم بالأخر باللغة العامية السعودية وما إلى ذلك.

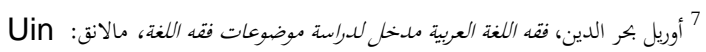
Malang Press
في المعاملات الرسمية وفي تدوين الشعر والنثر

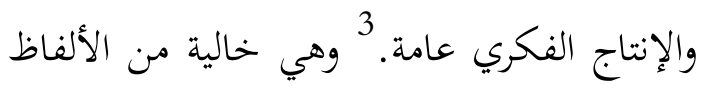
العامية أو السوقية. واللغة العامية هي اللغة اليومية. وهي لغة التخاطب في السوق والبيت. 4 أي لغة التخاطب غير الرسمي. ويتخذ مصطلحات العامية اسماءا كثيرة عند بعض اللغويين المحدثين ك "اللغة العامية" و "الشكل اللغوي الدارج" و "اللغة المحكية" و "العربية الدارجة" و "الكلام الدارج" و و "الكلام

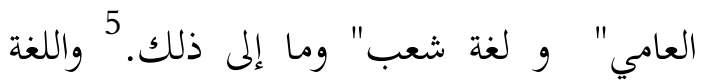
العامية التي يستخدمها العرب متنوعة بين المناطق، منها العامية للعراقي والعامية لليمني والعامية

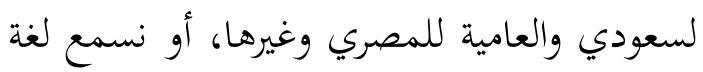
هذيل، لغة تيم ولغة طيئ التي جائت في المعجمات العربية لايريدون بها سوى ما نعنيه الأن

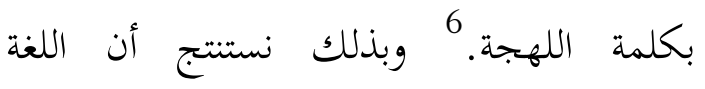
الفصحى هي لهجة، المثال اللهجة لبني سعد، لبني

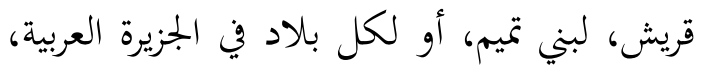
ولكن تستخدم في حالة رسمية. ذهب نولدكه Noldeke اللهجات الأساسية في الجزيرة العربية كالحجاز ونجد وإقليم الفرات، لأن الاختلافات بين هذه اللهجات كنت قليلة، وتبعه جويدي Guidi في الزعم بأن الفصحى ليست لهجة معينة لقبيلة

3 ت نفس المرجع, ص: 147. 4 يُّة عفيف الدين دمياطي، محاضرة في علم اللغة الاجتماعي، جومبامج: حقوق الطبع محفوظة للمؤلف، 2009 ص: 72 5 5 نفس المرجع، ص: 72. 6 حاتم صالح الضامن، فقه اللغة، القاهرة: دار الأفاق العربية 2007 ص:53 
صار نطقه "ده"، وهناك أيضا تحذيف الصوت الأول أي حذفت هزة القطع للتصال بالكلمة بعدها. فصارت تلك الكلمة نطقت بـ دَكْثر. وبذلك، ينبغي لنا كعاشق الأغاني الأجنبية أن نعلم اختلاف التعبير في صوت الحروف المتماثل في اللغة الفصحى واللغة العامية المصرية التي تسبب التغير في الصائتة والصامتة. وبهذا، أرادت الباحثة أن تعرف الفرق بين اللغة الفصحى واللغة العامية المصرية من الناحية الصوتية في هذا الغناء بالتوسع. فلذلك اختارت الباحثة موضوع " تحليل التقابل بين اللغة الفصحى واللغة العامية المصرية من الناحية الصوتية في غناء عمرو دياب" واختارت الباحثة غناء عمرو دياب لأن في هذا الغناء كثيرا من التبادل الأصوات وحذف وزيادتا و والمفردات والقواعد التي تختلف بالفصحى.

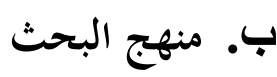

واستخدمت الباحثة في هذا البحث المنهج

النوعي ونوعه هو الدراسة المكتبية. ومصادر البيانات في هذا البحث هي غناء عمرو دياب

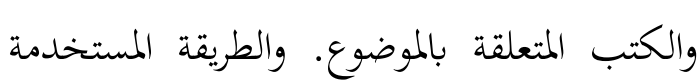
في جمع البيانات هي التأريخ والوثائق، وأما المنهج المستخدم في تحليل البيانات هو المنهج الوصفي بطريقة تحليل التقابل بين اللغة الفصحى واللغة العامية المصرية من الناحية الصوتية. وتستخدم

December 2015):

https://doi.org/10.15408/a.v2i2.2184.
وكل لغة يشتمل على الصوت المرتب

بالكلمة والجملة. وكذلك الأغاني الموسقية العربية المركبة على المزيج الطويل. وبنائها في مكان التدرج الذي يتكون من كلمات فكلمات وعبارات فعبارات حتى من صف فصف. إذا بدل أو غيّر الصوت في اللغة المعينة يسبب تغيير المعنى المعينة أيضا. هذا كما عرفنا في اللغة الفصحى، ولكن قد بد لا يسبب تغيير المعنى من الكلمة ولو بتبديل الصوت أو تغييره في اللغة العامية المصرية. مثل غناء ويّاه في كلمة ده أكثر، أها في اللغة الفصحى هي هذا أكثر. ووصف من تلك الكلمة فهو: ده اسم إشارة في اللغة العامية المصرية مستخدم للمفرد للمذكر. والاسم الإشارة المستخدم في اللغة العامية المصرية يتكون من: ده،

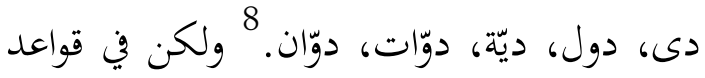
اللغة الفصحى، أسماء الإشارة وهي: ذا للمفرد مذكر، وذان وذين للمثنى المذكر وذه وتهللمفرد

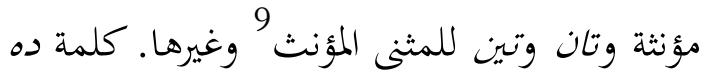
فيها تبادل الصامتين بين الذال والدال. والذال

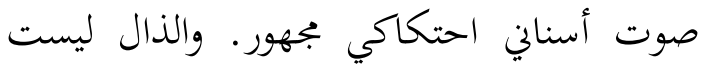

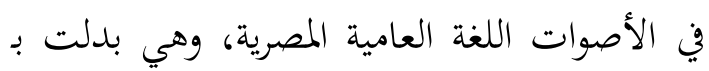

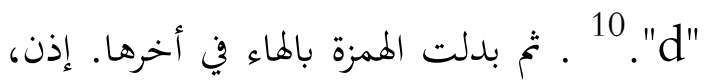

${ }^{8}$ Basmah Al-Makkiyah and Rizki Febrian AlBanjari, Percakapan Bahasa Amiyah Mesir Sehari-Hari, 2nd ed. (Mesir: Unit Penerbitan Karya Keluarga Mahasiswa Kalimantan Mesir, 2009), 9.

9 الشيخ مصطفى الغلاييني جامع الدبوس اللغة العربية، القاهرة- مصر: المكتبة التوفيقية، الطبعة الثالثة، 2013، ص: 86

10 Mufrodi, 'Fonologi dan Morfologi Bahasa Arab 'Amiyah Mesir', Arabiyat: Jurnal Pendidikan Bahasa Arab dan Kebahasaaraban 2, no. 2 (31 
والمصدر الثنائي هو المصدر المتناول من

المراجع المكتبة التي تتعلق بالموضوع. أي الكتب

التي تتعلق باللغة الفصحى واللغة العامية المصرية من الناحية الصوتية. مثلا كتاب:

1. حسام البنساوي، العربية الفصحى ولهجاتا،

القاهرة: مكتبة الثقافة الدينية، 2002

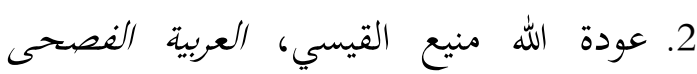

(مرونتها وعقلانيتها وأسباب خودها) عمان:

$$
\text { دار البداية، الطبعة الأولى، } 2008
$$

3. عُمَّم عالي الخولي، معجمر علم الأصوات،

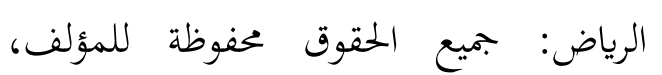

الطبعة الأولى 1982

4. علي عبد الواحد وافي، فقه اللغة، القاهرة:

$$
\text { لجنة البيان العربي، } 1962
$$

5. كمال تُحَّل بشر، الأصوات العببية، القاهرة:

$$
\text { مكتبة الشباب، } 1990
$$

6. إسوة أدريانا، تحليل التقابل والأخطاء، باميكاسان: الجامعة الإسلامية الحكومية

$$
\text { باميكاسان، } 2015
$$

7. Basmah Al-Makkiyah dan Rizki Febrian Al-Banjari Percakapan Bahasa Amiyah Mesir Sehari-hari, Unit Penerbitan Karya Keluarga Mahasiswa Kalimantan Mesir (KMKM), Kairo, 2009.

8. Mufrodi, Fonologi dan Morfologi Ammiyah Mesir, Arabiyat, Jurnal Pendidkan Bahasa Arab dan Kebahasaan, 2, (2), 2015, 192215.
الباحثة في هذا البحث مصدرين، وهما المصدر الأساسي والمصدر الثنائي. المصدر الأساسي هو المصدر الذي يكصل مباشرة من موضع البحث بكيفية أخذ البيانات مباشرة في الموضوع كموضوع البيانات المطلوب. وأما لمصدر الأساسي في هذا البحث فهو نص غناء عمرو دياب "ويّاه"، ويمكن للباحثة وصف السمات أو التمييزات بين اللغتين ألا وهما اللغة الفصحى واللغة العامية المصرية من جهة صوتية. وكلمة غناء عمرو دياب كما يلي: ده اللي كان نفسي فيه لو تيجي صدفة بتمعني فرصة عمري اضياعها ليه مش معقول عيني قدام عنيه ده اكثر من اللي حلمت بيه جه اليوم اللي انا مستانيه علشان اقول وياه الحياة هتحلي وانا معاه هو ده اللي انا باتمناه

$$
\text { وايوه هقي قلبي عيني شيفاه احساس انه احلى واغلى الناس وعيد ما هو بقى جانبي و مش بعيد اهدي بقى لاقيناه }
$$
فرحة قالبي كأنه عيد مستنيه مش هاستني اليوم كمان لازم اقوله انا من زمان عيني عليه. 
من من الصوت المهموس. والأصوات المهموسة في اللغة العربية كما ينطقها بجيدوا القراءات اليوم وكما ينطقها

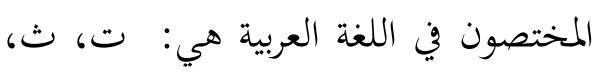
ح، خ، س، ش، ص، ط، ف، ق، ك) هـ. ولكن قد يقال أن الأصوات المهموسة عشرة، يجمعها "فحثه شخص سكت فئ والصوت المجهور. والأصوات المجهورة في اللغة العربية كما ننطقها اليوم هي: بك، ج، د، ذ، ر، ز، ض، ظ، ع، غ، ل، م، ن. والفرق بين الهمس والجهر هي أن الجهر مظهره الصوت وأن الهمس مظهره النفس. ب. الأصوات الصامتة بحسب مواضع النطق أو

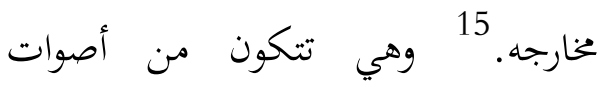
شفوية، وهي صوت يشترك في نطقه الشفة

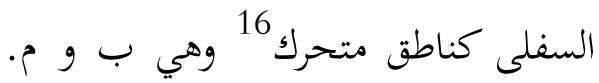
وأسنانية شفوية هي ف، وأسنانية أو

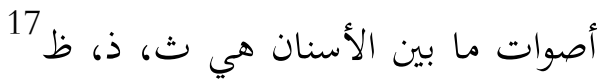
وأسنانية لثوية هي في اللغة العربية هي:

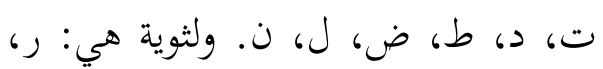
س، ز، ص. ولثوية حنكية هي: هي ج، ش. وأصوات وسط الحنك هي: ري.

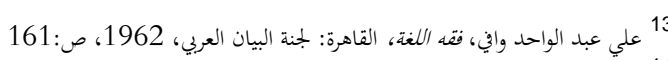

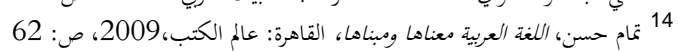
15 عبد الوهاب رشيدي، علم الأصوات النطقي (نظرية ومتارنة مع تطبيق في القرأن

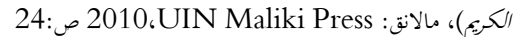
16 مُحمّ عالي الخولي، معجم عالقم الأصوات، الرياض: جميع الحقوق محفوظة للمؤلف، الطبعة الأولى 1982 صالي الخول: 86

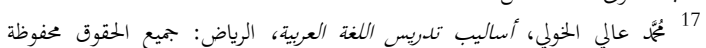
للمؤلف، 1989، ص:39
وطريقة جمع البيانات التي احتاجتها الباجثة

فاستخدمت طريقة جعها بالتأريخ والوثائق الموجودة في نص غناء عمرو دياب، والتأريخ والوثائق الموجودة في المراجع المكتبة بكيفية دوام قراءة البيانات ودراسة المراجع المتعلقة وكتابة

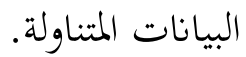

\section{ج. الأصوات العامية المصرية التي تكون في غناء عمور دياب}

قبلما بحثت الباحثة عن الأصوات في اللغة العامية المصرية التي تكون في غناء عمرو دياب فينبغي للباحثة بحث اللغة الفصحى للمقارنة بينها

\section{1. الأصوات في اللغة الفصحى}

والأصوات في اللغة الفصحى هي تتكون من أصوات الصامتة وتسمى بالحروف عند علماء العربية تختلف من لغة إلى أخرى، ولكن درجة الاختلاف هنا أقل من درجة الاختلاف بين اللغات في حالة الحركات 11 . والقاعدة الاختلات العامة على كل حال هي تقسيم الأصوات الصامتة إلى ثلاثة تقسيمات رئيسة وهي: أ. الأصوات الصامتة بحسب وضع الأوتار

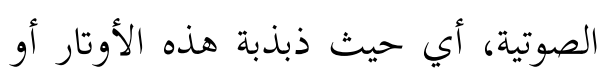

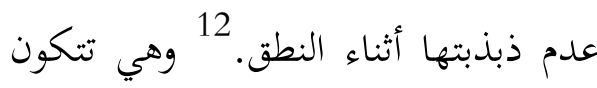
11 إسوة أدريانا، علم الأصوات، الجامعة الاسلامية الحكومية باميكاسان، 2015، ص: 12 12 15

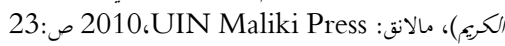


الفتحة والضمة والكسرة. وثلاثة طوال وهي ألف المد وواو المد وياء المد.

2. الأصوات العامية المصرية التي تكون في غناء

\section{عمرو دياب}

وأما الأصوات اللغة العامية المصرية التي

تكون في غناء عمرو دياب كما يلي:

أ. الأصوات الصامتة أو الساكنة

الأصوات الصامتة في اللغة العامية

المصرية مثابتة من الأصوات الصامتة في اللغة

الفصحى في الحقيقة، في الأصوات الصامتة

بحسب وضع الأوتار كانت أم بحسب

مخارجها أم بحسب صفة الصوت. وتشتمل

الأصوات الصامتة التي تكون في غناء عمرو

دياب على أ ب ت "ج (g)" ح خ د "ذ

(د)" ر ز س ش ص ض ع ع ف ف "ق

(ء)" لك ل م ن و ي. ولكن الأصوات اللغة رن صن

العامية المصرية التي تكون في غناء عمرو

دياب أصوات لا نجدها في اللغة الفصحى

منها:

1) صوت لثوي حنكي مركب مجهور هو

الجيم. 22 والجيم ليست في الأصوات اللغة

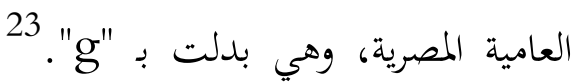

وهذا الصوت يكون في كلمة غناء عمرو

دياب تيجي التي نطقت بـ tigi، تجمعني

التي نطقت بـ tigma’ni، جه التي

22 عبد الوهاب رشيدي، علم الأصوات النطقي (نظرية ومقارنة مع تطبيق في القرأن

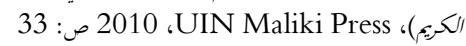

${ }^{23}$ Mufrodi, 'Fonologi dan Morfologi Bahasa Arab 'Amiyah Mesir', 195.
وأقصى الحنك هي: خُ، غن، ك، وأن 18

وأصوات لهوية هي ق. وأصوات حلقية

هي: ع، ح. وأصوات حنجرية هي: ء و

ج. الأصوات الصامتة بحسب صفة الصوت أو

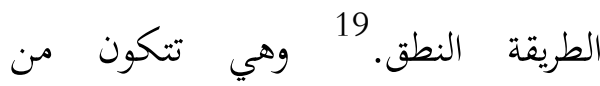

الأصوات الانفجارية وتسمى الوقفات.

والأصوات الانفجارية في اللغة الغربية هي:

ب6، ت، د، ض، ط، لك، ق،

والأصات الاحتكاكية. والأصوات

الاحتكاكية غي اللغة العربية هي: ف،

ث، ذ، ظ، ز، س، ص، ش، ح، غن، ع،

خ، هـ. وصوت انفجاري احتكاكي أو

مركب في اللغة العلابية هي: ج. وصوت

مكرر، فهي في معظم اللغات مكررة أو

مترددية 20 وهو ر. وصوت وصوت جانبي، وهو

ل. . والأصوات الأنفية، هي م، ن. ن.

وأنصاف الحركة في اللغة العربية هي و،

ي

وتتكون الأصوات في اللغة الفصحي من

أصوات الصائتة. وعرف العلماء الأصوات

الصائتة بأنه صوت بجهور لايسمع عند النتائج

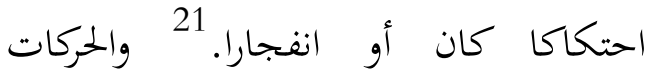

الأساسية في اللغة العربية ثلاثة قصار هي

18 كمال لمهَّ بشر، الأصوات العربية، القاهرة: مكتبة الشباب، 1990 ص: 90 (19 19 20 أحمد مختار عمر، أسس علم اللغة، القاهرة: عالم الكتب، الطبعة الثامنة 1968 صل:

21 26 عبد الوهاب رشيدي، علم الأصوات النطقي (نظرية ومقارنة مع تطبيق في القرأن الكريم)، مالانق: 2010،UIN Maliki Press ص: 27 
قصيرة، والفتحة والضمة والكسرة كلها طويلة، صوت "ف" وتسمى بالحفضة وهي

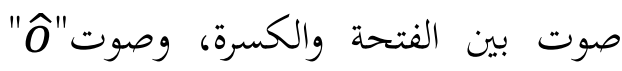
وتسمى بالرفعة وهي صوت بين الفتحة والضمة.واللغة العامية المصرية في غناء عمرو دياب تبادل الأصوت وزيادة الأصوات وتحذيف الأصوات وغيرها أو كما قيل بتفاعل أصوات الكلمة بعضها ببعض. 1) تبادل الأصوات، ويطرأ التبادل بين الأصوات الصامتة كان أم بين الأصوات

الصائتة

أ) (التبادل بين الصائتين

لا تهتم اللغة العامية المصرية

القواعد النحوية ويغلب في استخدام

إعراب جزم كل الحروف في أخر

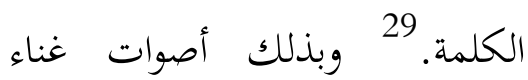

عمرو دياب تتكون من جزم كل

الحروف في أخر الكلمة، ولو كان

أصل كلمات غنائه يتكون من حركة

الفتحة والضمة والكسرة التي بدلت

من السكون. وتلك الأصوات تشمل

على: كانَ، صدفة، اليوزٌ، الناسن،

هو (تبادل الفتحة إلى السكون).

معتول، أكثر، قدازم، أقولْ، وياهُ،

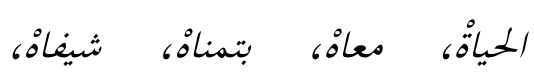

نطقت بـ gih وجانبي التي نطقت بـ

gambi

2) صوت أسناني احتكاكي مجهور هو

الذال. 24 والذال ليست في الأصوات

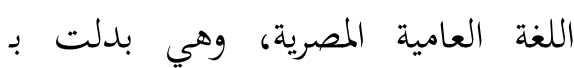

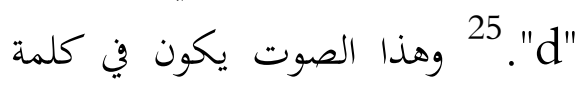

غناء عمرو دياب ده أصلها هي هذا.

3) صوت لهوي انفجاري مهموس هو

القاف. 26 وخرج القاف انتقل إلى الحلق

"باحثا عن أقرب الأصوات شبها به من

الناحية الصوتية" فتعمق القاف في الحلق

عند المصريين. 27 إذن، القاف ليست فئين

الأصوات اللغة العامية المصرية، وهي

بدلت بـ "ع". 28 وهذا الصوت يكون في الاصغية

كلمة غناء عمرو دياب معتول نطقت

بـ مأول، قدام نطقت بـ أدام، أقول

نطقت بـأوْل، قلبي نطقت بـ ألبي، يقولي

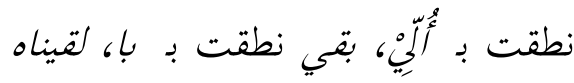

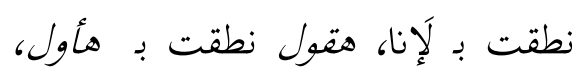

أقوله نطقت بـ أُوْلا.

ب. الأصوات الصائتة أو اللين أو الحركة

والحركات الأساسية في اللغة العامية

المصرية هي الفتحة والضمة والكسرة كلها

24

25 Mufrodi, 'Fonologi dan Morfologi Bahasa Arab 'Amiyah Mesir', 195.

26 عبد الوهاب رشيدي، المرجع السابق، ص: 42 27 رمضان عبد التواب، بجوث ومقالات في اللغة، القاهرة: مكتبة الخانجي بالقاهرة،

${ }^{28}$ Mufrodi, 'Fonologi dan Morfologi Bahasa Arab 'Amiyah Mesir', 195. 
صفات كليهما. 32 كما وقع في كلمة

غناء هعتول فإن حرف العين و

حرف الهمزة يمتزجان أي يتقاربان في

مخرجهما أي في الحلقية فصارت

مأول وهناك صوت صائت مركب

بدل إلى صوت صامت مناسب

بالحركة قبله، فيسبب صوتا ثالث

يتضمن على ذلك الصوتان

المتقاربين.

وغناء عمرو دياب فيه تبادل

الصائت الطويل إلى الصائت القصير،

مثل كان نغسي نطقت بـ كن

نضسي، عمري أضيعها نطقت بـ عمَر

ضَبَّعها، من اللي حملت نطق بـ مِن

لَحلمت، ده اللي انا نطقت بـ دَلَّنا،

$$
\text { أققول نطقت بـ أنَّا . }
$$

ب) التبادل بين الصامتين

في اللغة العامية المصرية تبادل

الصامت بالصامت وهو صوت

لثوي حنكي مركب بجهور هو الجيم.

والجيم ليست في الأصوات اللغة

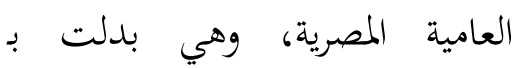

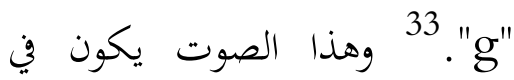

كلمة غناء عمرو دياب تيجي التي

نطقت بـ tigi، تجمعني التي نطقت
أحساسن، لقيناه، ععيذ، فرحةٌ، لازمْ

(تبادل الفتحة إلى السكون). دهة

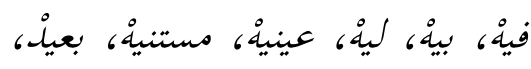

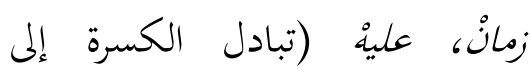

السكون). تيجيك، تبجمعني (تبادل

الضمة إلى السكون). صُدفة (تبادل

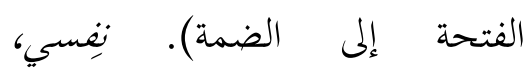

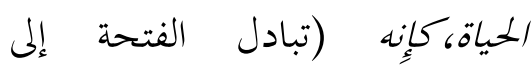

الكسرة).

وفي غناء عمرو دياب فيه

صوت الالتصاق بين الصائتين بعد

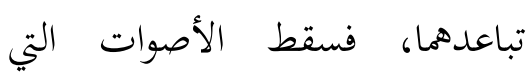

تفصلهما ويتكون منهما صوت

صامت مركب. 30 وتلك الأصوات

تشتمل على ليه نطقت بـ leh، عليه

نطقت بـ aleh، (صوت الرفعة)

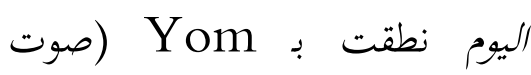

الحفضة). أو كما عرفنا أن الياء

بصوت "ف " تسمى بالحفضة وهي

صوت بين الفتحة والكسرة، والواو

بصوت"

صوت بين الفتحة والضمة. 31 ومن وصن

تجاور الصوتان المختلفان

مخارجهما هو يمتزجان معا. فيتكون

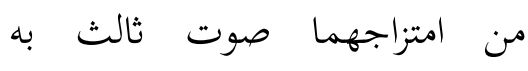

30 إسوة أدريانا، علم الأصوات، الجامعة الإسلامية الحكومية باميكاسان،2015، ص:

31 Mufrodi, 'Fonologi dan Morfologi Bahasa Arab 'Amiyah Mesir', 195.

'Amiyah Mesir', 195. 
المستقبل. 37 كما وقع في كلمة غناء

هتحلي أصلها ستحلي، هتول أصلها سأقول، هاستني أصلها ساستني.

$$
\text { 3) تحذيف الصوت }
$$

في اللغة العامية المصرية تحذيف الصوت، في أول الكلمة ك يا أختي نطق يَنْتي، أي حذف الهمزة في أول الكلمة. ووقع هذا التحذيف في وسط الكلمة مثل: كاتب نطق بـ كتُب وكذلك في أخر الكلمة، المثال: شيفهُ نطق بـ شيفا. 38 وهذا يجري أيضا في غناء عمرو دياب الذي يشتمل على تحذيف الصوت في أول الكلمة أُضِيَعِها نطقت بـ ضَيَّعْها، ده

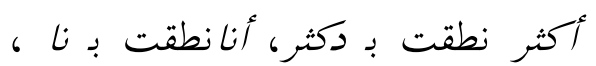

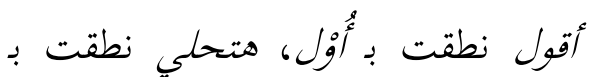

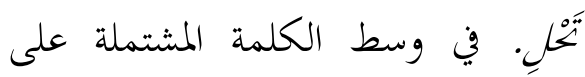

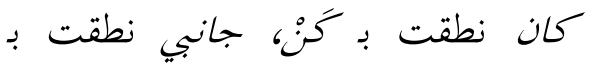
جَنبي، في أخر الكلمة المشتملة على ده نطقت بـ د، جِه نطقت بـ جه، الحياة نطقت بـ الحيا، شيغاه نطقت بـ شيغا،

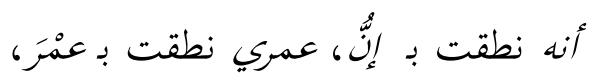
عيني نطقت بـ عينِ، خلي نطقت بـ خلِّ، بقي نطقت بـ با، احلى نطقت بـ بـ بـ احل.

37 Al-Makkiyah and Al-Banjari, Percakapan Bahasa Amiyah Mesir Sehari-Hari, 10.

${ }^{38}$ Mufrodi, 'Fonologi dan Morfologi Bahasa Arab 'Amiyah Mesir', 203.
بـ tigma'ni، جه التي نطقت بـ gih وجانبي التي نطقت بـ gambit احتكاكي مجهور هو الذال. والذال ليست في الأصوات اللغة العامية المصرية، وهي بدلت بـ "d". 34 وهذا الصوت يكون في كلمة غناء عمرو دياب ده أصلها هي هذا. وصوات لهوي انفجاري مهموس هو القاف. والقاف ليست في الأصوات اللغة

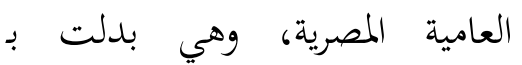
"ء". 35 وهذا الصوت يكون في كلمة وهرية بلدية غناء عمرو دياب معقول نطقت بـ مأول، قدام نطقت بـ أدام، أقول نطقت بـ أوْل، قلبي نطقت بـ ألبي، يقولي ألِّي، بقي نطقت بـ بـ با، لقيناه نطقت بـ لإِنا، هقول نطقت بـ بـ هأول، أقوله نطقت بـ أُوْلا

$$
\text { 2) زيادة الصوت }
$$

في اللغة العامية المصرية زيادة الصوت مثل الباء في وزن فعل تفعل الذي غير بـ اتفعل. وهناك زيادة الألف. والباء دليلة الوقت الحال أو الأن. 36 كما ولئ الدها. وقع في كلمة غناء بتمناه أصلها تمناه. وزيادة الهاء في أول الكلمة دليلة الوقت

${ }^{34}$ Mufrodi, 195. 
4) القواعد النحوية المختلفة بالفصحى يأيت المعنى غالبا جاريا على القواعد المتعارف عليها. 39 وقد عرفنا أن اللغة العامية المصرية لها القواعد النحوية مع اختلاف باللغة الفصحى. مثل: الاسم الإشارة في قواعد اللغة الفصحى يتكون من: ذا، ذه، ذان، ذين، تان، تين وغيرها. ولكن الاسم الإشارة المستخدم في اللغة العامية المصرية تتكون من: ده، 40 دى، دول، ديّة، دوّات، د دوّان. وكذلك في اسم موصول الذي بدلت بـ اللي. وغناء عمرو دياب يتكون من الاسم الإشارة ده وهو في اللغة الفصحى هذا والاسم الموصول اللي وهو في اللغة

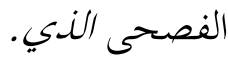

5) في اختيار المفردات المختلفة بالفصحى اختلفت المفردات ي اللغة واللغة العامية المصرية غالبا، مثل: كلمة مشْ بمعنى غير في اللغة الفصحى. وفي اللغة العامية المصرية للدليل إلى شيى سبي فزيادة كلمة "مش" في أول الكلمة. 41 وغناء عمرو فئي دياب يتكون من مش وب وليه. 


\section{د. اختلافات في اللغة العامية المصرية من الناحية الصوتية

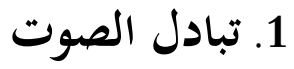

أ. أتبادل بين الصائتين

\begin{tabular}{|c|c|c|c|c|c|}
\hline وصفت & وصف الصوامت & تبادل الصائتين & اللغة الفصحى & اللغة العامية & 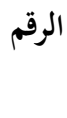 \\
\hline الفتحة القصيرة: & "ن "ن" "ن"، وضعها في أسنانية لثوية كذلك وضعها في الشفوية، و وضعها في لثوية، و "و" & الفتحة الصائتة/a/ إلى السكون & كانَ، صدفةَ، اليومَ، & كانْ، صدفةُ، اليومُ، الناسْ، & 1 \\
\hline حركة مرتفعة القصيرة: & 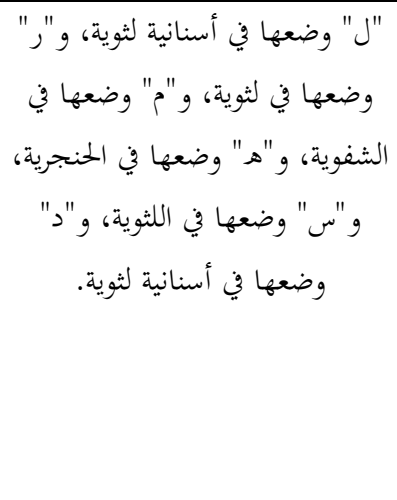 & الضمة الصائتة /ull إلى السكون & 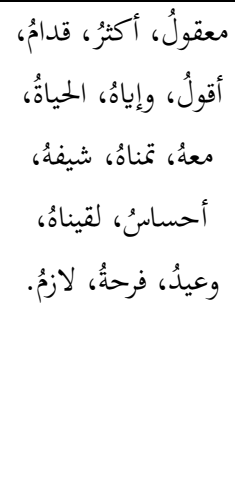 & 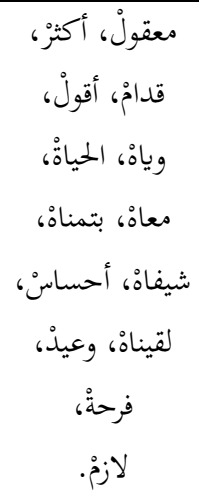 & 2 \\
\hline الكسرة القصيرة: & "هـ" وضعها في الحنجرية، و"د" وضعها في أسنانية لثوية، و"ن" & الكسرة الصائتة |i إلى السكون & هذا، فيدِه، بهِ، إليهِ، & 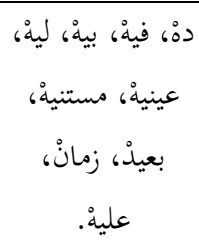 & 3 \\
\hline الضمة القصيرة & "ت" وضعها في أسنانية ثثوية & الضمسة الصائتة إلى & تُوجي، بُجمعني. & تِيجي، تِّمَعني. & 4 \\
\hline الفتحة القصيرة الضمة & "ص" وضعها في لثوية & الفتحة الصائتة & صَدفة & صُدفة & 5 \\
\hline الفتحة القصيرة الكسرة & "ن" وضعها في أسنانية لثوية. "ا" & الفتحة الصائتة الكسرة & نَفَسي، الحَحياة، كأنَّهُ & كَفِسي، إلِحياة، & 6 \\
\hline
\end{tabular}

ب. صوت مركب (الرفعة والخفضة)

\begin{tabular}{|c|c|c|c|c|}
\hline صوت الحفظة & صوت الرفعة & اللغة الفصحى & اللغة العامية المصرية & الرقم \\
\hline & $|\hat{e}| \leftarrow$ /ai $/$ & لماذا، عليه & ليه، عليه & 1 \\
\hline $\mid \hat{o} / \leftarrow / \mathrm{ao} /$ & & اليوم إم & اليوم ل & 2 \\
\hline
\end{tabular}


ج. التبادل بين الصائت الطويل إلى الصائت القصير

\begin{tabular}{|c|c|c|c|c|}
\hline الوصف & التبادل & اللغة الفصحى & المصرية العامية & الرقم \\
\hline 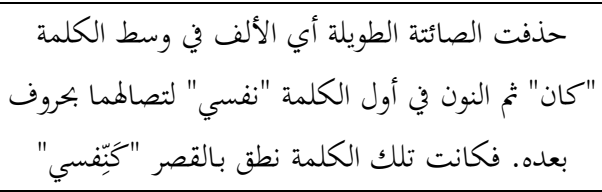 & 111 & كانَ نَسْي & كانْ ِِفسي & 1 \\
\hline 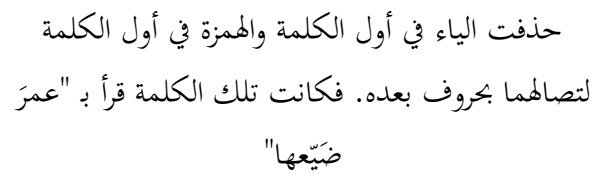 & |ي| & عمرِيْ أُضِيَعها & عمري اضيعها & 2 \\
\hline 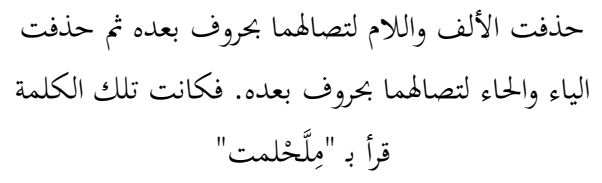 & 111 & مِن التي حملت & مِنْ اللّي حْملت & 3 \\
\hline 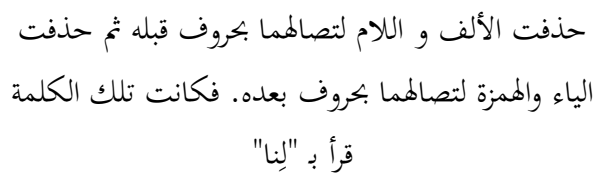 & |ili g و & التي أنا & اللي انا & 4 \\
\hline حذفت الهاء و الألف واللام ثخ حذفت الياء والهمزة & 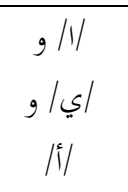 & هذه التي أنا & دة اللي انا & 5 \\
\hline حذفت الألف اللينة والواو والهمزة فكانت تلك الكلمة & |il & أقول أنا & اقول انا & 6 \\
\hline
\end{tabular}

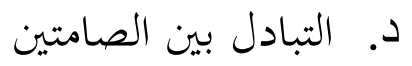

\begin{tabular}{|c|c|c|c|c|}
\hline الوصف الصامت & التبادل & اللغة الفصحى & اللغة العامية & الرقم \\
\hline الجيم صوت لثوي حنكي & ج ج إلى g & 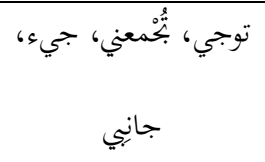 & تيجي، تبِمْعني، جه، & 1 \\
\hline مهموس والممزة صوت حنجرية & ق إلى أ & قلبي، يقول، بقي، قدام، أقول، & يقول، بقي، لقيناه، أقوله & 2 \\
\hline
\end{tabular}




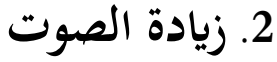

\begin{tabular}{|c|c|c|c|c|}
\hline الوصف & 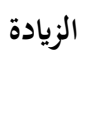 & اللغة الفصحى & المعة العامية & 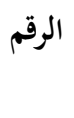 \\
\hline زيادة حرف الباء في أول الفعل الماضي أو الفعل المضارع في اللغة & | & تمناه & بتمناه & 1 \\
\hline الهاء بدل من سين، وهي بمعنى مستقبل & هـ & ستحلي & هتحلي & 2 \\
\hline
\end{tabular}

3. تحذيف الصوت

أ. تحذيف الصوت في أول الكلمة

\begin{tabular}{|c|c|c|c|c|}
\hline الوصف & تحذيف & اللغة الفصحى & اللغة العامية المصرية & 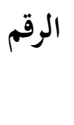 \\
\hline حذفت الهمزة لتصالها بحروف قبله. فكانت تلك & |í & أُضِيِعُهَا & اضَيَُّْها & 1 \\
\hline حذفت الهمزة لتصالهما بحروف قبله. فكانت تلك & |íl & هذا أكثر & ده اكثَر & 2 \\
\hline حذفت الممزة لتصالهما بحروف قبله. فكانت تلك & |í| & أنا & انكا & 3 \\
\hline حذفت الهمزة لتصالها بحروف قبله. فكانت تلك & |íl| & أقول & اقول & 4 \\
\hline حذفت الهاء لتصالمما بحروف قبله. فكانت تلك & |هـ| & ستحلي & هتحلي & 5 \\
\hline
\end{tabular}

ب. تحذيف الصوت في وسط الكلمة

\begin{tabular}{|c|c|c|c|c|}
\hline الوصف & 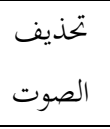 & اللغة الفصحى & اللغة العامية المصرية & الرقم \\
\hline حذفت الألف في وسط الكلمة. فكانت تلك & 111 & كانَ & كانْ & 1 \\
\hline حذفت الألف في وسط الكلمة. فكانت تلك & 111 & جاني & جانبي & 2 \\
\hline
\end{tabular}




\begin{tabular}{|c|c|c|c|c|}
\hline الوصف & تحذيف & اللغة الفصحى & اللغة العامية المصرية & 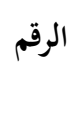 \\
\hline حذفت الهاء في أخر الكلمة. فكانت تلك & $|ه|$ & هذا، جيء، الحياة & شها & 1 \\
\hline 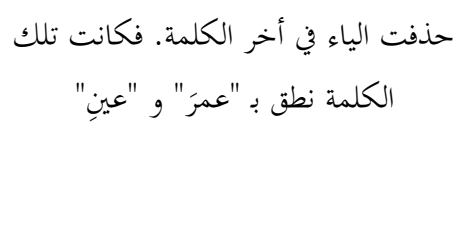 & |ي| - ل اي| & عيني، خلى، عمري، & عيني، خلي، بقي عمرى، & 2 \\
\hline حذفت الممزة في أخر الكلمة. فكانت تلك & 111 & أحلي & احلى & 3 \\
\hline
\end{tabular}

4. القواعد النحوية

\begin{tabular}{|c|c|c|c|}
\hline الوصف & اللغة الفصحى & اللغة العامية المصرية & الرقم \\
\hline اسم الموصول في اللغة العامية المصرية يتكون من اللي سواء للمذكر & التي/الذي & اللي الي & 1 \\
\hline اسم الإشارة في اللغة العامية المصرية يتكون من ده الدال على & هذا & ده & 2 \\
\hline
\end{tabular}

\begin{tabular}{|c|c|c|c|c|}
\hline الوصف & صوت المخفردات & اللغة الفصحى & اللغة العامية & 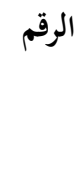 \\
\hline اللي أصلها اسم الإشارة "التي" في اللغة الفصحى & ت ت ت إلى ل & التي & 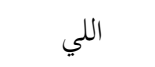 & 1 \\
\hline دون، لا بدل من الكلمة السلبية، منها غير، ليس، وفي هذه الكلمة، الهاء بدلا من & غير إلى مش & غير & 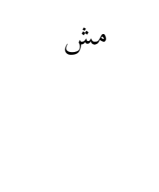 & 2 \\
\hline ل ليه بدل من لماذا & لماذا إلى ليه & 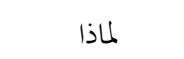 & ليه & 3 \\
\hline
\end{tabular}


ه. تشابه اللغة العامية المصرية باللغة الفصحى في غناء عمرو دياب

\begin{tabular}{|c|c|c|c|}
\hline الوصف & 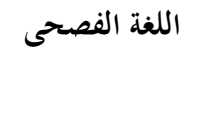 & المغة العامية & 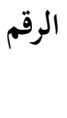 \\
\hline متشابه في المفردات والمعنى وفي الأصوات كان في الصامت والصائت & لو & لو & 1 \\
\hline متشابه في المفردات والمعنى وفي الأصوات كان في الصامت والصائت وفي & مِن "حرف جر" & من "حرف جر" & 2 \\
\hline متشابه في المفردات والمعنى وفي الأصوات كان في الصامت والصائت وفي & و "حرف العطف" & و العطف" & 3 \\
\hline متشابه في المفردات والمعنى وفي الأصوات كان في الصامت والصائت & 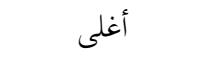 & أغلى & 5 \\
\hline متشابه في المفردات والمعنى وفي الأصوات كان في الصامت والصائت & أحلى & أحلى & 6 \\
\hline متشابه في المفردات والمعنى وفي الأصوات كان في الصامت والصائت & خلاص & خلاص & 7 \\
\hline متشابه في المفردات والمعنى وفي الأصوات كان في الصامت والصائت وفي & L & L & 8 \\
\hline
\end{tabular}

الناسن، هؤ(التبادل من الفتحة إلى السكون)،

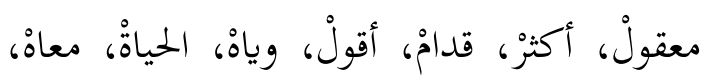

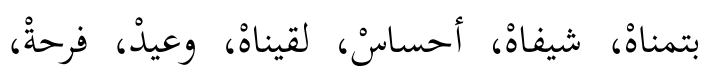

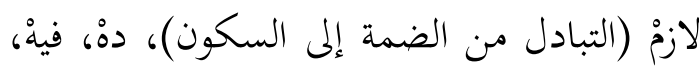

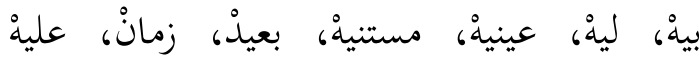

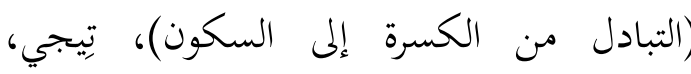

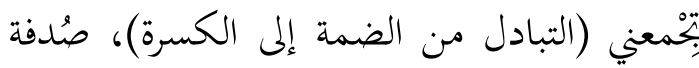
(التبادل من الفتحة الضمة)، نِفسي، إلحياة، كإنه

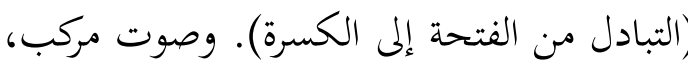

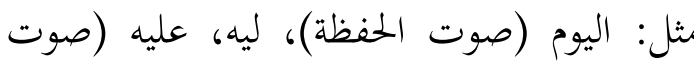
الرفعة). وتبادل الصائت الطويل إلى الصائت

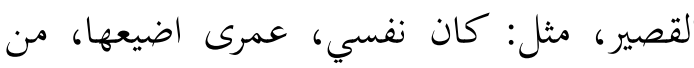

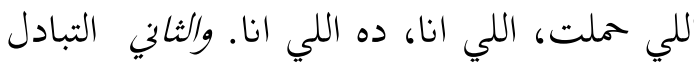
بين الصامتين، مثل: تيجي، بتمعني، جه، جانبي

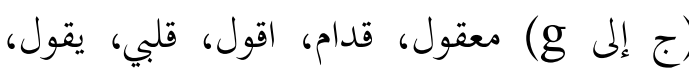
بقي، لقيناه، أقوله (ق إلى ء).
و. التنبؤ عن المشكلات التي تنشأ عند فهم اللغة العامية المصرية من الناحية الصوتية في غناء عمرو دياب بعدما عرضت الباحثة عن الأصوات العامية المصرية التي تكون في غناء عمرو دياب ووجه اختلافتها وتشابها من اللغة الفصحى، لفئل

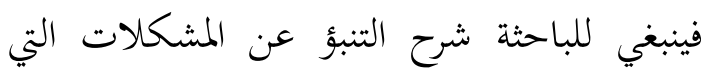

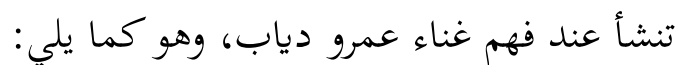
قد عرفنا أن الطلاب الإندونيسي يشعرون بالصعبة في فهم الأفلام والإذاعات والإخبار وغيرها في اللغة العامية المصرية لأفم غير عيادة للتكلم والسمع باللغة العامية المصرية حتى كانوا يشعرون ذلك. وفي غناء عمرو دياب "وياه" تغيير الأصوات المختلف باللغة الفصحى الذي يتكون من تبادل الصوت الذي يشتمل على: الأول

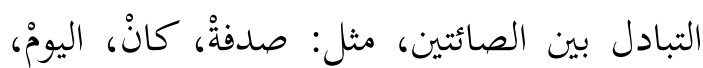




\section{References}

\section{قائمة المراجع و المصادر اللغة العربي}

إبراهيم، عبد علي، المجه الفني لمدرسي اللغة

العربية، الطبعة السابعة عشرة، القاهرة:

$$
\text { دار المعارف، دون السنة. }
$$

أدريانا، إسوة، تحليل التقابل والأخطاء، باميكاسان: الجامعة الإسلامية الحكومية

$$
\text { باميكاسان، }
$$

أدريانا، إسوة، علم الأصوات، الجامعة الاسلامية

$$
\text { الحكومية باميكاسان، } 2015 .
$$

بحر الدين، أوريل فقه اللغة العربية مدخل لدراسة موضوعات فقه اللغة، مالانق: Uin

\section{9 ،Malang Press}

البنساوي، حسام، العربية الفصحى ولجاته،

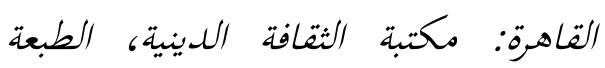

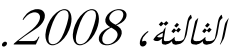

الخولي، يُمَّهُ عالي، معتجم علم الأصوات، الرياض:

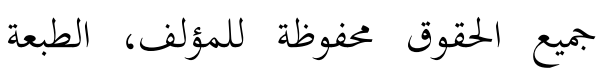

$$
\text { الأولى، } 1982 .
$$

رشيدي، عبد الوهاب، علم الأصوات النطقي (نظرية ومقارنة مع تطبيق في القرأن

، UIN Maliki Press الكريم) .2010

الزيدي، كاصد، فقه اللغة، عمان: دار الفرقان

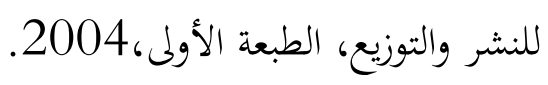

ويتكون أيضا غناء عمرو دياب من زيادة

الصوت، مثل: بتمناه (ب)، هتحلي، هاقول،

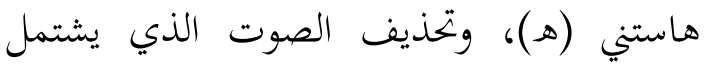

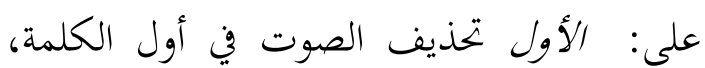
مثل: اضيعها، ده اكثر، انا، اقول (حذف ()، هتحلي (حذف ه). والثاني تحذيف الصوت في

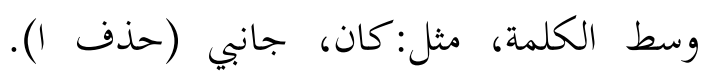
والثالث تحذيف الصوت في أخر الكلمة، مثل: ده، جه، الحياة، شيفاه، انه (حذف ه))، عمري، عيني، خلي، بقي (حذف ي). ويتكون أيضا غناء عمرو دياب من من بن القواعد النحوية تختلف بالفصحى، مثل: ده أها في اللغة الفصحى هي هذا، واللي أها في اللغة الفصحى هي التي. وكذلك يتكون من المفردات المختلفة بالفصحى، مثل: مُشْْ بمعنى غير، وليه أها في اللغة الفصحى هي لماذا. فالطلاب الذين بالين

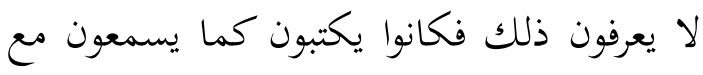
أن الأصوات التي يسمعون في غناء عمرو دياب له تغيير واختلاف باللغة الفصحى. واللغة الفصحى قرأت كما تكتب وكتبت كما تقرأ وهذا لايجري في اللغة العامية المصرية لأن هناك اختلافات كثيرة من الناحية الصوتية بالفصحى. وغناء عمرو دياب فيه التشابه باللغة الفصحى،

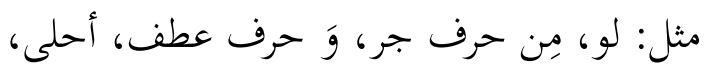

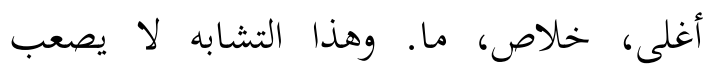
الطلاب للفهم عنها لأها دون تغيير الأصوات في الفاله الصامت أم في الصائت، أو دون تغيير المفردات أو اختلاف القواعد النحوية. 
صالح الضامن، حاتم، فقه اللغة، القاهرة: دار مذكور، علي أحمد، تلديس فنون اللغة العببية،

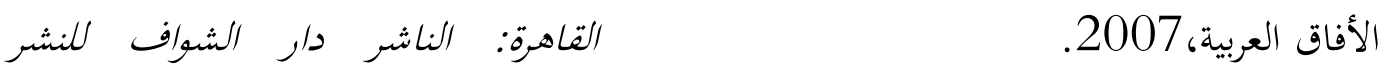

$$
\text { والتوزيع، } 1991 .
$$

يعقوب، إميل بديع، فقه اللغة العربية وخصائصها،

بيروت: دار الثقافة الاسلامية، 2011.

\section{قائمة المراجع و المصادر اللغة الإندونيسية}

Al-Makkiyah, Basmah, and Rizki Febrian Al-Banjari. Percakapan Bahasa Amiyah Mesir Sehari-Hari. 2nd ed. Mesir: Unit Penerbitan Karya Keluarga Mahasiswa Kalimantan Mesir, 2009.

Mufrodi. 'Fonologi dan Morfologi Bahasa Arab 'Amiyah Mesir'. Arabiyat: Jurnal Pendidikan Bahasa Arab dan Kebahasaaraban 2, no. 2 (31 December 2015). https://doi.org/10.15408/a.v2i2.21 84.

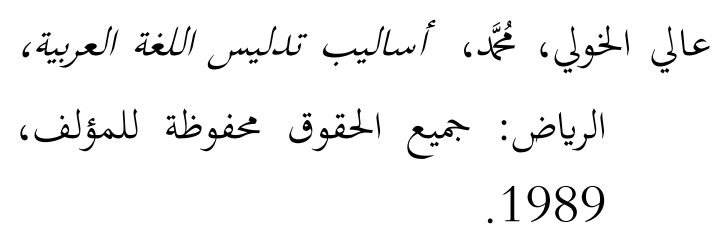
مكتبة الشباب، 1990 . 
